\title{
Inverse barocaloric effect in the giant magnetocaloric La-Fe-Si-Co compound
}

\author{
Lluís Mañosa', David González-Alonso', Antoni Planes', Maria Barrio², Josep-Lluís Tamarit², Ivan S. Titov³, \\ Mehmet Acet $^{3}$, Amitava Bhattacharyya ${ }^{4} \&$ Subham Majumdar ${ }^{4}$
}

Application of hydrostatic pressure under adiabatic conditions causes a change in temperature in any substance. This effect is known as the barocaloric effect and the vast majority of materials heat up when adiabatically squeezed, and they cool down when pressure is released (conventional barocaloric effect). There are, however, materials exhibiting an inverse barocaloric effect: they cool when pressure is applied, and they warm when it is released. Materials exhibiting the inverse barocaloric effect are rather uncommon. Here we report an inverse barocaloric effect in the intermetallic compound $\mathrm{La}-\mathrm{Fe}-\mathrm{Co}-\mathrm{Si}$, which is one of the most promising candidates for magnetic refrigeration through its giant magnetocaloric effect. We have found that application of a pressure of only $1 \mathrm{kbar}$ causes a temperature change of about $1.5 \mathrm{~K}$. This value is larger than the magnetocaloric effect in this compound for magnetic fields that are available with permanent magnets.

\footnotetext{
${ }^{1}$ Departament d'Estructura i Constituents de la Matèria. Facultat de Física. Universitat de Barcelona. Diagonal 645. Barcelona Knowledge Campus (BKC), 08028 Barcelona, Catalonia, Spain. ${ }^{2}$ Departament de Física i Enginyeria Nuclear. ETSEIB. Universitat Politècnica de Catalunya. Diagonal 647 and Center for Research in NanoEngineering, Pascual i Vila, 15. Barcelona Knowledge Campus (BKC), 08028 Barcelona, Catalonia, Spain. ${ }^{3}$ Experimentalphysik, Universität Duisburg-Essen, D-47048 Duisburg, Germany. ${ }^{4}$ Department of Solid State Physics. Indian Association for the Cultivation of Science. Jadavpur, Kolkata 700 032, India. Correspondence and requests for materials should be addressed to L.M. (email: Iluis@ecm.ub.es).
} 
$\mathrm{T}$ he need for environmentally friendly cooling technologies has boosted research in solid-state refrigerant materials. In most cases, refrigeration relies on the temperature change caused by the adiabatic application (or removal) of an external field (caloric effects). Under normal circumstances, temperature changes are small, but when the material is close to a phase transition, moderate variations in the external field can provoke temperature changes of several degrees and give rise to so-called giant caloric effects. The tuning parameter for the caloric effect (external field) can be any generalized thermodynamic force, provided that the change in the conjugated generalized thermodynamic displacement is large enough. Hence, giant caloric effects have already been reported for magnetic field (magnetocaloric effect $)^{1-3}$, electric field (electrocaloric effect) ${ }^{4,5}$, uniaxial stress (elastocaloric effect) ${ }^{6}$ and hydrostatic pressure (barocaloric effect) $)^{7,8}$.

In a recent work ${ }^{7}$, we postulated that most giant magnetocaloric materials would also exhibit large barocaloric effects. This claim is also supported by recent theoretical studies ${ }^{9,10}$. Here we report on the barocaloric effect of the giant magnetocaloric material $\mathrm{LaFe}_{11.33} \mathrm{Co}_{0.47} \mathrm{Si}_{1.2}$. $\mathrm{La}(\mathrm{Fe}, \mathrm{Si})_{13}$, and the derived quaternary compounds are considered nowadays to be the most promising working materials for magnetic refrigeration technology ${ }^{11,12}$. They feature relatively large entropy changes with a very low hysteresis and do not contain toxic materials. Materials with reduced hysteresis are highly desirable because irreversibilites associated with such a hysteresis cause a reduction in the refrigerant capacity ${ }^{13,14}$. $\mathrm{LaFe}_{13-x} \mathrm{Si}_{x}$ compounds are stable with the cubic $\mathrm{NaZn}_{13}$ structure (space group $F m \overline{3} c$ ) in a concentration range $1.2 \leq x \leq 2.5$ (ref. 15). Below the Curie temperature $T_{C}$ in the range $200-260 \mathrm{~K}$, the compound orders ferromagnetically, and above $T_{C}$ an itinerant-electron metamagnetic transition is induced by an external field ${ }^{16}$. Such an itinerant-electron metamagnetic transition originates from a magnetic-field-induced change in the density of states of the $3 \mathrm{~d}$ electrons at the Fermi level ${ }^{17}$. A strong magnetovolume effect originates from the existence of a peak in the electronic density of states, close to the Fermi level, that yields a negative contribution from spin fluctuations to the magnetostriction, resulting in a volume increase on cooling from the paramagnetic to the ferromagnetic state. Two alternative methods have been reported to bring the transition temperature to values close to room temperature without significant modification in the magnetocaloric properties ${ }^{11}$. One method is to add interstitial hydrogen that expands the crystalline lattice and modifies the exchange interaction between iron atoms. Another method is to replace some Fe atoms by other magnetic transition metals. It has been found that replacing Fe by only a few percent of Co enables a fine tuning of the critical temperature, and it has already been possible to produce industrially meaningful quantities (kilograms) of $\mathrm{La}-\mathrm{Fe}-\mathrm{Co}-\mathrm{Si}$ material with good magnetocaloric properties around room temperature ${ }^{18}$. The paramagnetic to ferromagnetic transition in $\mathrm{La}-\mathrm{Fe}-\mathrm{Si}$ (and Co-doped) compounds is accompanied by a sizeable volume change of about $1 \%$ (ref. 19). There is no change in the crystal symmetry, but the sample isotropically expands as it transforms from the high-temperature paramagnetic to the lowtemperature ferromagnetic phase. Such a volume change makes the compound highly sensitive to the application of an external hydrostatic pressure; and indeed the effect of pressure on the magnetic and magnetocaloric properties has been reported ${ }^{16,20}$. The volume change reported for $\mathrm{La}-\mathrm{Fe}-\mathrm{Si}$ is one of the largest for magnetocaloric materials undergoing magnetostructural transitions. Therefore, this compound is an excellent candidate for exhibiting large barocaloric effects associated with its magnetostructural transition.

We have used calorimetry under hydrostatic pressure to obtain the pressure-induced entropy change, and direct temperature measurements to obtain the adiabatic temperature change. These are the two key parameters characterizing a caloric effect. The results are complemented by the measurement of the magnetic field-induced

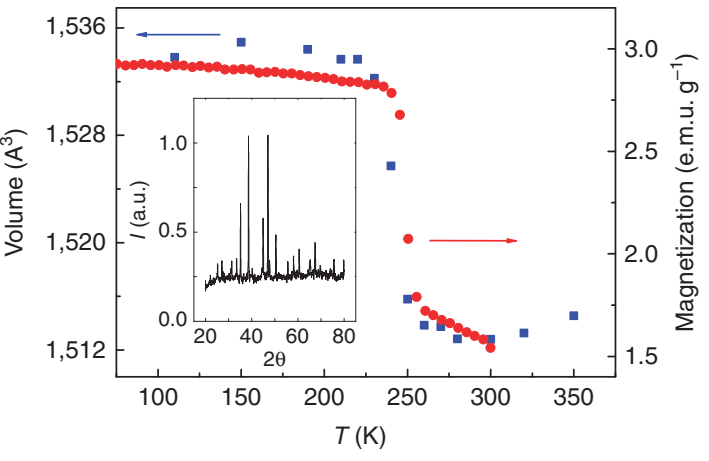

Figure 1 | Temperature dependence of the volume and the magnetization in $\mathbf{L a F e}_{\mathbf{1 1 . 3 3}} \mathrm{Co}_{\mathbf{0 . 4 7}} \mathbf{S i}_{\mathbf{1 . 2}}$. Unit cell volume (squares) and low-field magnetization (circles) as a function of temperature. Inset: room temperature $\mathrm{X}$-ray diffraction pattern. The peak at $\sim 5^{\circ}$ corresponds to $\alpha$-iron.

temperature and entropy changes associated with the magnetocaloric effect. The giant barocaloric effect for the material is inverse: that is, we find that the sample warms when pressure is adiabatically released (the entropy decreases under isothermal conditions). The magnetocaloric effect is conventional (the sample warms up when magnetic field is applied and cools down when it is removed).

\section{Results}

Structural and magnetic characterization. The sample studied in this investigation is a polycrystalline $\mathrm{LaFe}_{11.33} \mathrm{Co}_{0.47} \mathrm{Si}_{1.2}$ alloy. $\mathrm{X}$-ray diffraction data (inset in Fig. 1) confirm that at room temperature the alloy crystallizes in the $\mathrm{NaZn}_{13}$ structure (space group $F m \overline{3} c$ ) with a lattice parameter of $11.48 \AA$. When cooled in the absence of an external field, the sample orders ferromagnetically below a temperature $T_{C} \sim 250 \mathrm{~K}$ with a concomitant isotropic expansion in the unit cell volume of about $1 \%$, as illustrated in Figure 1 . The most widely used method to compute the entropy change associated with a field-induced caloric effect is from isothermal measurements of the generalized displacement vs force curves. Although this method turns out to be very convenient for determining the magnetocaloric effect, where the magnetization can be measured to high precision using superconducting quantum interference device magnetometry, the method is not readily applicable for determining the barocaloric effect because the involved volume changes are small, and they cannot be measured easily with enough precision. An alternative method is to measure directly the heat exchanged when the transition is thermally induced under an external constant field. It requires the use of purpose-built calorimetric devices, and it has been shown to provide reliable results for various caloric effects.

Caloric effects. Examples of calorimetric curves at selected values of hydrostatic pressure are shown in the inset of Figure 2. The curves correspond to heating runs, and have been corrected for the baseline drift. An endothermic feature associated with the ferromagnetic-paramagnetic transition is clearly observed. The transition shifts towards lower temperatures as the pressure is increased. This is consistent with pressure enhancing the stability of the lower volume phase, which is the high-temperature phase. The computed entropy changes (integrated entropy curves) at selected values of the hydrostatic pressure are shown in Figure 2 (details of the computation can be found on Methods section). On heating, the entropy increases by $11.4 \mathrm{Jkg}^{-1} \mathrm{~K}^{-1}$. It must be taken into account that the base-line correction subtracts a background entropy increase, so that the measured increase in entropy is solely due to the occurrence of the phase transition. Numerical subtraction of the integrated entropy curves 




Figure 2 | Entropy as a function of temperature in $\mathrm{LaFe}_{11.33} \mathrm{Co}_{\mathbf{0 . 4 7}} \mathrm{Si}_{1.2}$. Entropy values, referred to the value at ambient temperature, for selected values of hydrostatic pressure. The inset shows calorimetric curves at selected values of the hydrostatic pressure. Curves appear noisy owing to the weakness of the detected signal, associated with the small mass of the sample. Black lines correspond to $0 \mathrm{kbar}$; green, $0.8 \mathrm{kbar}$; magenta, $1 \mathrm{kbar}$; cyan, $1.2 \mathrm{kbar}$; blue, $1.4 \mathrm{kbar}$; orange, $1.7 \mathrm{kbar}$ and olive, $2.1 \mathrm{kbar}$.

renders the pressure-induced entropy change (barocaloric effect) at each temperature. Results are shown in Figure 3a. The most relevant feature is the positive values of the entropy change, which corresponds to the uncommon inverse barocaloric effect. The magnitude of the barocaloric effect increases with increasing pressures, and a value of $8.6 \mathrm{Jkg}^{-1} \mathrm{~K}^{-1}$ is obtained for $p=2095$ bar. This value is $75 \%$ of the maximum expected value corresponding to the whole transition entropy change. Although the required pressures are larger than typical pressures in domestic fridges (based on compression of a cryogenic gas), they are still readily accessible by conventional hydraulic technologies.

In the studied sample, both magnetocaloric and barocaloric effects originate from the same physical mechanism: the strong magnetovolume interplay occuring at the para-ferromagnetic phase transition that encompasses concomitant changes in magnetization and volume. Therefore, it is interesting to compare the barocaloric with the magnetocaloric properties. To this end, we have measured isothermal magnetization curves at selected values of the temperature from which we have numerically computed the entropy change associated with the magnetocaloric effect. The results are consistent with data reported for alloys with compositions close to the studied sample ${ }^{21}$. The magnetocaloric effect is conventional (that is, decrease in entropy on applying a magnetic field), and the magnetic field-induced entropy change at $5 \mathrm{~T}\left(-9.2 \mathrm{Jkg}^{-1} \mathrm{~K}^{-1}\right)$ amounts to $\sim 80 \%$ of the whole transition entropy change.

Although entropy change is a good quantity to characterize caloric effects, any practical application requires knowledge of the adiabatic temperature change. Direct measurements of the adiabatic temperature change when pressure is rapidly released are shown in Figure $3 \mathrm{~b}$ for pressures of 1 and $2 \mathrm{kbar}$. The first evident result is the confirmation of the inverse character of the barocaloric effect: the sample warms when pressure is released. The temperature dependence of $\Delta T$ is in good agreement with that of $\Delta S$ (Fig. 3a). The adiabatic temperature change can be estimated from isothermal entropy data using $\Delta T \simeq-(T / C) \Delta S$, where $C$ is the heat capacity. Although a correct evaluation requires measuring $C$ at different values of $p$, a rough estimation using $C_{p}$ data measured at atmospheric pressure (inset in Fig. 4) shows that the measured values of $\Delta T$ are lower than the estimated ones. This difference must be mainly ascribed to a lack of perfect adiabatic conditions in the measurements under pressure due to changes in sample surroundings. Therefore, the actual
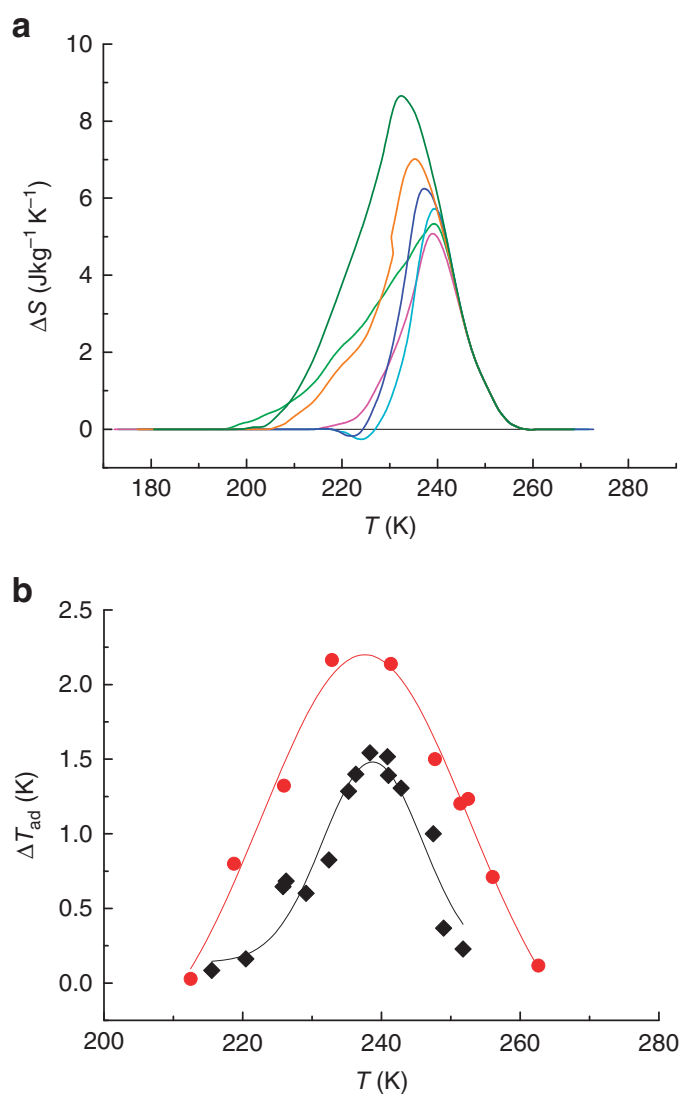

Figure 3 | Barocaloric effects in $\mathrm{LaFe}_{11.33} \mathrm{Co}_{\mathbf{0 . 4 7}} \mathrm{Si}_{\mathbf{1 . 2}}$. (a) Isothermal entropy change as a function of temperature associated with the isothermal application of selected hydrostatic pressures. Black lines correspond to $0 \mathrm{kbar}$; green, $0.8 \mathrm{kbar}$; magenta, $1 \mathrm{kbar}$; cyan, $1.2 \mathrm{kbar}$; blue, $1.4 \mathrm{kbar}$; orange, $1.7 \mathrm{kbar}$ and olive, $2.1 \mathrm{kbar}$. (b)Adiabatic temperature change on fast release of hydrostatic pressure. Diamonds correspond to $1 \mathrm{kbar}$ and circles to $2 \mathrm{kbar}$ (lines are guides to the eye).

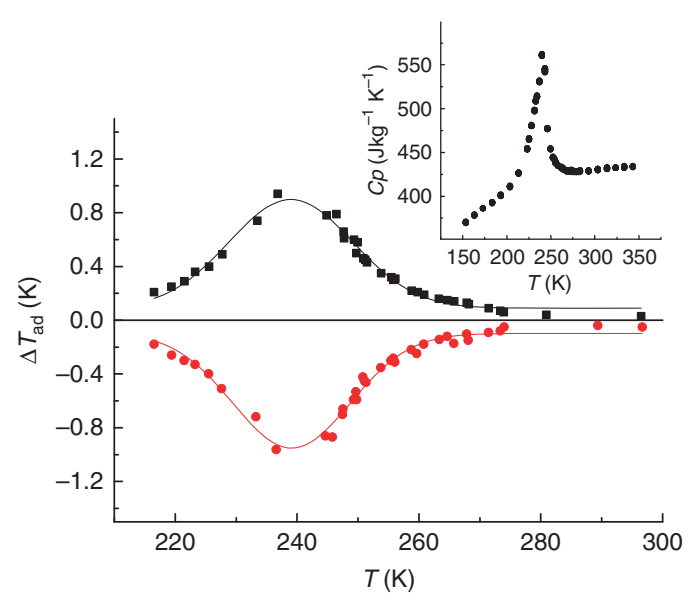

Figure 4 | Magnetocaloric effect in $\mathrm{LaFe}_{11.33} \mathrm{Co}_{\mathbf{0 . 4 7}} \mathrm{Si}_{1.2}$. Adiabatic temperature measurements on applying ( $\Delta T^{\text {on }}$, squares) and removing ( $\Delta T^{\text {off }}$, circles) a 1T magnetic field. Lines are guides to the eye. The inset shows the temperature dependence of the heat capacity at atmospheric pressure and in the absence of magnetic field.

temperature change is expected to be larger than the data presented in Figure 3b. For comparison we have also directly measured the temperature change associated with the application, $\Delta T^{\text {on }}$, and 
removal, $\Delta T^{\text {off }}$, of a magnetic field of $1 \mathrm{~T}$ (Fig. 4). The excellent coincidence of $\left|\Delta T^{\mathrm{on}}\right|$ and $\left|\Delta T^{\mathrm{off}}\right|$ is an indication of the negligible irreversible losses for this compound. As a consequence of the good adiabatic conditions in magnetic measurements and low hysteretic losses, the values estimated for $\Delta T$ agree fairly well with measured ones.

\section{Discussion}

In $\mathrm{La}-\mathrm{Fe}-\mathrm{Si}$ (and doped compounds), the total entropy is due to the magnetic contribution from the itinerant $3 d$ electrons and a contribution from the lattice (the electronic contribution from the $s p$ conduction electrons is smaller). It has been established that hydrostatic pressure affects both contributions: on the one hand, pressure increases the width of the effective $3 d$ bands, thus opposing to the onset of the ordered magnetic state. On the other hand, lattice contribution is also affected by pressure through a strong magnetoelastic coupling that modifies phonon frequencies, which can be expressed in terms of a renormalized Debye temperature ${ }^{9}$.

In systems undergoing a phase transition involving interplay between different material properties (volume and magnetization in the case of present study), caloric effects associated with each of these properties may be of opposite sign. Actually, in the absence of frustration effects, the primary property providing the dominant change in entropy at the transition should be associated with a conventional caloric effect whereas secondary properties may provide conventional or inverse effects depending on specific features of their coupling to the primary property. For instance, on cooling, $\mathrm{Ni}-\mathrm{Mn}$-In magnetic shape-memory alloys undergo a structural transition which involves changes of symmetry and volume and is accompanied by a decrease of the magnetization. Therefore, in this material, the barocaloric effect must be conventional. The decrease in magnetization is a consequence of the interplay of magnetism and structure and originates from the antiferromagnetism associated with the change of distances between $\mathrm{Mn}$ atoms induced by the structural transition. This feature explains the well-known inverse magnetocaloric effect in these materials ${ }^{22}$. By contrast, in the $\mathrm{La}-\mathrm{Fe}-\mathrm{Si}$ compounds, the transition is essentially of magnetic nature, and, therefore, the magnetocaloric effect is conventional. Because spin fluctuations induce a volume increase at the transition, the barocaloric effect in this material is inverse.

A good parameter to assess the usefulness of a given material for refrigeration purposes is the relative cooling power (RCP) that quantifies the energy absorbed in a refrigerating cycle. A suitable estimation of RCP is provided by the area below the $\Delta S$ vs $T$ curve (see Fig. 3a). The computed values for the magnetocaloric $\left(45 \mathrm{Jkg}^{-1} \mathrm{~T}^{-1}\right)$ and barocaloric $\left(90 \mathrm{Jkg}^{-1} \mathrm{kbar}^{-1}\right)$ effects compare well with data for the best giant magnetocaloric materials. Moreover, although magnetic fields stronger than $1 \mathrm{~T}$ are difficult to implement, pressures much larger than those used here can be applied (using clamp cells, for instance), and, therefore, the barocaloric effect appears more advantageous because RCP is expected to substantially increase, as a result of a much broader peak resulting from the shift in the transition temperature.

In summary, we have identified the occurrence of the barocaloric effect in one of the technologically most promising giant magnetocaloric materials. The barocaloric effect has been found to be inverse, and direct temperature measurements prove that the material heats up on removal of hydrostatic pressure. The magnitude of the barocaloric effect for moderate pressures is larger than the magnetocaloric effect for magnetic fields reachable with permanent magnets, and, therefore, the reported effect is potentially useful for eco-friendly solid-state refrigeration purposes. Finally, it is worth mentioning that the inverse barocaloric effect arises from the volume increase on cooling through the phase transition. Such a volume increase gives rise to a negative thermal expansion (NTE) within a certain temperature range. Materials exhibiting colossal NTE resulting from phase transitions are receiving considerable interest ${ }^{23}$. From thermodynamic arguments, it can be expected that these materials with NTE will also exhibit inverse barocaloric effects.

\section{Methods}

Thermodynamic background. For any material subjected to hydrostatic pressure $(p)$, magnetic field $(H)$ and temperature $(T)$ changes, the general expression for an entropy change reads

$$
\mathrm{d} S=\frac{C}{T} \mathrm{~d} T+\left(\frac{\partial M}{\partial T}\right)_{p, H} \mathrm{~d} H-\beta v \mathrm{~d} p
$$

where we have used the Maxwell relations:

$$
\left(\frac{\partial S}{\partial H}\right)_{p, T}=\left(\frac{\partial M}{\partial T}\right)_{p, H},\left(\frac{\partial S}{\partial p}\right)_{H, T}=-\left(\frac{\partial v}{\partial T}\right)_{p, H}=-\beta v
$$

where $\beta$ is the thermal expansion.

This expression shows that large entropy changes are expected when either volume or magnetization change drastically with temperature under constant external $H$ and $p$. This situation typically occurs in the vicinity of phase transitions.

The sign of the entropy change is determined by the temperature derivatives of $M$ and $v$. At constant pressure, application of a magnetic field will reduce entropy in those systems for which magnetization decreases with increasing temperature. As this is the most commonly found situation, this effect is termed as conventional magnetocaloric effect. By contrast, in those materials where magnetization increases with increasing temperature, application of a magnetic field will increase the entropy, resulting in an inverse magnetocaloric effect. The equivalent reasoning also holds for hydrostatic pressure: materials for which volume increases with increasing temperature give rise to a conventional barocaloric effect (entropy decreases with application of pressure) whereas those with a decreasing volume with increasing temperature show an inverse barocaloric effect.

It is also worth noticing that at constant magnetic field, materials with negative thermal expansion $(\beta)$ will exhibit inverse barocaloric effect (equation (1)).

Experimental details. Ingots were prepared by arc melting the pure elements under argon atmosphere in the stoichiometric ratio. The composition was determined by energy-dispersive $\mathrm{X}$-ray photoluminiscence analysis to correspond to $\mathrm{LaFe}_{11.33} \mathrm{Co}_{0.47} \mathrm{Si}_{1.2}$. The sample was annealed at $1,323 \mathrm{~K}$ for 15 days and quenched. Room temperature powder X-ray diffraction patterns were recorded using a Bruker AXS diffractometer ( $\mathrm{Cu} \mathrm{K} \alpha$ radiation, $2 \theta$ range from $20^{\circ}$ to $80^{\circ}$ with a step size of $0.02^{\circ}$ ). Temperature-dependent high-resolution X-ray powder diffraction measurements were performed on a transmission mode diffractometer using Debye-Scherrer geometry equipped with horizontally mounted INEL cylindrical position sensitive detectors containing 4,096 channels $\left(0.029^{\circ} 2 \theta\right.$ step $)$ and by using monochromatic $\mathrm{Cu} \mathrm{K} \alpha$ radiation. Temperature was controlled by means of a liquid nitrogen 700 series Oxford Cryostem Cooler. The heating rate between the measurements was $1.3 \mathrm{~K} \mathrm{~min}^{-1}$ and, before data collection, the sample temperature was stabilized for $15 \mathrm{~min}$.

Magnetization measurements were carried out using a superconducting quantum interference device magnetometer. From these data, the magneticfield-induced entropy change was numerically computed, taking into account the procedures described in (ref. 24), to avoid undesired overestimated values. Specific heat was measured using a modulated differential scanning calorimeter.

Hydrostatic pressure calorimetry was carried out in a purpose-built calorimeter described in (ref. 7). From calorimetric curves at selected values of the hydrostatic pressure $p$, the entropy change (referenced to a given state at $T_{0}$ ) is computed as:

$$
S(T, p)-S\left(T_{0}, p\right)=\int_{T_{0}}^{T} \frac{1}{T} \frac{\dot{Q}(p)}{\dot{T}} \mathrm{~d} T,
$$

where $\dot{Q}(p)$ is the heat flux and $\dot{T}$ is the heating/cooling rate.

For direct adiabatic temperature measurements, a chromel-alumel thermocouple was embedded into the sample. In pressure measurements, the sample was placed into the calorimeter and it was first cooled down under applied pressure to a temperature low enough to ensure that the whole sample was in the low-temperature phase. Then, it was heated (keeping the applied pressure) up to the measuring temperature. After thermalization, the pressure was rapidly released and the output of the thermocouple was recorded at a rate of $1 \mathrm{~Hz}$. This procedure was repeated for each measuring temperature. The effect of thermal losses to the surroundings was quantified by performing experiments at temperatures well above and well below the transition. For magnetic measurements, the device described in (ref. 25) was used. The sample was first brought to a temperature high enough to ensure that it was on the high-temperature phase. Then, it was cooled down to the desired temperature, allowed to thermalize, and magnetic field was rapidily applied (at a rate $1 \mathrm{Ts}^{-1}$ ) while the output of the thermocouple was recorded at a rate $0.5 \mathrm{~Hz}$. After $30 \mathrm{~s}$, the field was removed (at a rate $1 \mathrm{Ts}^{-1}$ ). This procedure was repeated for each measuring temperature. 


\section{References}

1. Pecharsky, V. K. \& Gschneidner, K. A. Jr. Giant Magnetocaloric effect in $\mathrm{Gd}_{5} \mathrm{Si}_{2} \mathrm{Ge}_{2}$. Phys. Rev. Lett. 78, 4494 (1997).

2. Tegus, O., Brück, E., Buschow, K. H. J. \& de Boer, F. R. Transition-metal-based magnetic refrigerants for room-temperature applications. Nature 415, 150-152 (2002).

3. Krenke, T. et al. Inverse magnetocaloric effect in ferromagnetic Ni-Mn-Sn alloys. Nat. Mater. 4, 450-454 (2005).

4. Mischenko, A. S., Zhang, Q., Scott, J. F., Whatmore, R. W. \& Mathur, N. D. Giant electrocaloric effect in thin-film $\mathrm{PbZr}_{0.95} \mathrm{Ti}_{0.05} \mathrm{O}_{3}$. Science 311, 1270-1271 (2006)

5. Neese, B. et al. Large electrocaloric effect in ferroelectric polymers near room temperature. Science 321, 821-823 (2008).

6. Bonnot, E., Romero, R., Mañosa, L., Vives, E. \& Planes, A. Elastocaloric effect associated with the martensitic transition in shape-memory alloys. Phys. Rev. Lett. 100, 125901 (2008).

7. Mañosa, L. et al. Giant solid-state barocaloric effect in the Ni-Mn-In magnetic shape-memory alloy. Nat. Mater. 9, 478-481 (2010).

8. Gorev, M., Bogdanov, E., Flerov, I. \& Laptash, N. Thermal expansion, phase diagrams and barocaloric effects in $\left(\mathrm{NH}_{4}\right)_{2} \mathrm{NbOF}_{5}$. J. Phys. Condens. Matter 22, 185901 (2010).

9. de Medeiros, L. G. , Jr.,, de Oliveira, N. A. \& Troper, A. Barocaloric and magnetocaloric effects in $\mathrm{La}\left(\mathrm{Fe}_{0.89} \mathrm{Si}_{0.11}\right)_{13}$. J. Appl. Phys. 103, 113909 (2008).

10. de Oliveira, N. A. \& von Ranke, P. J. Theoretical aspects of the magnetocaloric effect. Phys Rep. 489, 89-159 (2010).

11. Shen, B. G., Sun, J. R., Hu, F. X., Zhang, H. W. \& Cheng, Z. H. Recent progress in exploring magnetocaloric materials. Adv. Mater. 21, 4545-4564 (2009).

12. Gutfleisch, O. et al. Magnetic materials and devices for the 21st century: stronger, lighter, and more energy efficient. Adv. Mater. 23, 821-842 (2011).

13. Provenzano, V., Shapiro, A. J. \& Shull, R. D. Reduction of hysteresis losses in the magnetic refrigerant $\mathrm{Gd}_{2} \mathrm{Ge}_{2} \mathrm{Si}_{2}$. Nature 429, 853-857 (2004).

14. Shamberger, P. J. \& Ohuchi, F. S. Hysteresis of the martensitic phase transition in magnetocaloric-effect Ni-Mn-Sn alloys. Phys. Rev. B 79, 144407 (2009).

15. Palstra, T. T. M., Mydosh, J. A., Nieuwenhuys, G. J., van der Kraan, A. M. \& Buschow, K. J. Study of the critical-behaviour of the magnetization and electrical-resistivity in cubic $\mathrm{La}(\mathrm{Fe}, \mathrm{Si})_{13}$ compounds. J. Magn. Magn. Mater. 36, 290-296 (1983).

16. Fujita, A., Fujieda, S., Hasegawa, Y. \& Fukamichi, K. Itinerant-electron metamagnetic transition and large magnetocaloric effects in $\mathrm{La}\left(\mathrm{Fe}_{x} \mathrm{Si}_{1-x}\right)_{13}$ compounds and their hydrides. Phys. Rev. B 67, 104416 (2003).

17. Fujita, A., Fukamichi, K., Wang, J.- T. \& Kawazoe, Y. Large magnetovolume effects and band structure of itinerant-electron metamagnetic $\mathrm{La}\left(\mathrm{Fe}_{x} \mathrm{Si}_{1-x}\right)_{13}$ compounds. Phys. Rev. B 68, 104431 (2003).
18. Katter, M., Zellmann, V., Reppel, G. W. \& Uestuener, K. Magnetocaloric properties of $\mathrm{La}(\mathrm{Fe}, \mathrm{Co}, \mathrm{Si})_{13}$ bulk material prepared by powder metallurgy. IEEE Trans. Magn. 44, 3044 (2008).

19. Fujita, A., Fujieda, S., Fukamichi, K., Mitamura, H. \& Goto, T. Itinerantelectron metamagnetic transition and large magnetovolume effects in $\mathrm{La}\left(\mathrm{Fe}_{x} \mathrm{Si}_{1-x}\right)_{13}$ compounds. Phys. Rev. B 65, 014410 (2001).

20. Lyubina, J., Nenkov, K., Schultz, L. \& Gutfleisch, O. Multiple metamagnetic transitions in the magnetic refrigerant $\mathrm{La}(\mathrm{Fe}, \mathrm{Si}){ }_{13} \mathrm{H}_{x}$. Phys. Rev. Lett. 101, 177203 (2008).

21. Hu, F. et al. Very large magnetic entropy change near room temperature in $\mathrm{LaFe}_{11.2} \mathrm{Co}_{0.7} \mathrm{Si}_{1.1}$. Appl. Phys. Lett. 80, 826-828 (2002).

22. Planes, A., Mañosa, L. \& Acet, M. Magnetocaloric effect and its relation to shape-memory properties in ferromagnetic Heusler alloys. J. Phys.Condens. Matter 21, 233201 (2009)

23. Azuma, M. et al. Colossal negative thermal expansion in $\mathrm{BiNiO}_{3}$ induced by intermetallic charge transfer. Nat. Commun. 2, 347 (2011).

24. Caron, L. et al. On the determination of the magnetic entropy change in materials with first-order transitions. J. Magn. Magn. Mat. 321, 3559-3566 (2009).

25. Moya, X. et al. Cooling and heating by adiabatic magnetization in the $\mathrm{Ni}_{50} \mathrm{Mn}_{34} \mathrm{In}_{16}$ magnetic shape-memory alloy. Phys. Rev. B 75, 184412 (2007)

\section{Acknowledgements}

This work was supported by CICyT (Spain), projects MAT2010-15114, and FIS2008 00837 (Spain). Critical reading of the manuscript by N.D. Mathur and A. Saxena is acknowledged.

\section{Author contributions}

L.M. and A.P. planned the experiments in collaboration with J.T. and M.A. Sample preparation was carried out by A.B. and S.M. X-ray measurements were carried out by J.T., M.B., A.B. and S.M. Measurements in magnetic field were carried out by D.G., I.T. and M.A. Measurements under pressure were carried out by M.B. and J.T. All authors discussed the results and analysed the data. The manuscript was prepared by L.M. in collaboration with A.P., J.T., M.A. and S.M.

\section{Additional information}

Competing financial interests: The authors declare no competing financial interests.

Reprints and permission information is available online at http://npg.nature.com/ reprintsandpermissions/

How to cite this article: Mañosa, L. et al. Inverse barocaloric effect in the giant magnetocaloric La-Fe-Si-Co compound. Nat. Commun. 2:595 doi: $10.1038 /$ ncomms1606 (2011) 